\title{
A Method of Reducing Dimension of Space Variables in Multi-dimensional Black-Scholes Equations
}

\author{
${ }^{1}$ Hyong-chol O, ${ }^{2}$ Yong-hwa Ro ${ }^{3}$ Ning Wan \\ ${ }^{1,2}$ Faculty of Mathematics, Kim Il Sung University \\ Pyongyang, D. P. R. of Korea \\ ${ }^{3}$ Department of Applied Mathematics, Tong-ji University \\ Shanghai, China \\ e-mail: ${ }^{1}$ ohyongchol@yahoo.com, ${ }^{2}$ leewunghun@yahoo.com, ${ }^{3}$ blumping@etang.com
}

\begin{abstract}
We study a method of reducing space dimension in multi-dimensional Black-Scholes partial differential equations as well as in multi-dimensional parabolic equations. We prove that a multiplicative transformation of space variables in the Black-Scholes partial differential equation reserves the form of Black-Scholes partial differential equation and reduces the space dimension. We show that this transformation can reduce the number of sources of risks by two or more in some cases by giving remarks and several examples of financial pricing problems. We also present that the invariance of the form of Black-Scholes equations is based on the invariance of the form of parabolic equation under a change of variables with the linear combination of variables.
\end{abstract}

Keywords Black-Scholes equations; Multi-dimensional; Reducing dimension; Options; Foreign currency strike price; Basket option; Foreign currency option; Zero coupon bond derivative.

2010 Mathematics Subject Classification 35K15, 91B24.

\section{Introduction}

Multi-assets option prices satisfy multi-dimensional Black-Scholes partial differential equations [3. It is well known that the change of numeraire gives very important computational simplification in multi-assets option pricing. See [1]. But using the technique of numeraire change, we can reduce the number of sources of risks by one. See [4].

On the other hand, in some pricing problems of financial derivatives, we can see some transformations of variables reducing the number of sources of risks by two or more. For example, in [6], they reduced the number of sources of risks from 3 to 1 in pricing a European call foreign currency option. Their main method is to combine the change of numeraire and the transformation of multiplication of two variables. Furthermore in $[3]$ Jiang L.S reduced the number of sources of risks from $n$ to 1 at one try in pricing a basket option with the expiry payoff of the geometric mean of $n$ underlying assets without any use of change of numeraire.

The works [3, 6] make us confirm that there must be another general transformation (other than the change of numeraire) reducing the space dimension of multi-dimensional Black-Scholes partial differential equations and give us the clue of this article.

From the results of [3, 6] about pricing options with foreign currency strike price (pricing the option in Dollars), we have the idea that the transformation of multiplication of two variables would reserve the form of Black-Scholes partial differential equations. This transformation can reduce the dimension of space variable, and furthermore, this transformation can be applied repeatedly if some condition holds, so in such a case we can reduce the 
number of sources of risks by two or more. The result of Jiang [3] makes us to find more general transformation.

In this article we prove the invariance of the form of multi-dimensional Black-Scholes partial differential equations under the multiplicative transformation of variables and provide some examples where we can reduce the space dimension of multi-dimensional Black-Scholes partial differential equations with this transformation.

According to our study, in pricing financial derivatives, the possibility to reduce dimension depends on the expiry payoff function structures.

The remainder of this article consists as follows. In section 2 we prove the invariance of the form of multi-dimensional Black-Scholes partial differential equations under the multiplicative transformation of variables. In section 3 , we give such examples as

- Pricing options whose strike price is in a currency different from the stock price,

- Pricing a basket option with the expiry payoff of the geometric mean,

- Pricing a European call foreign currency option .

In section 4, we consider a relationship between the invariance of the form of BlackScholes partial differential equations and the invariance of the form of parabolic equations.

\section{The Invariance of the Form of Black-Scholes Equations}

Black-Scholes partial differential equations are one of main models in financial mathematics. The following partial differential equation

$$
\frac{\partial V}{\partial t}+\frac{1}{2} \sum_{i, j=0}^{n} a_{i j} S_{i} S_{j} \frac{\partial^{2} V}{\partial S_{i} \partial S_{j}}+\sum_{i=0}^{n}\left(r-q_{i}\right) S_{i} \frac{\partial V}{\partial S_{i}}-r V=0,
$$

is called the $(n+1)$-dimensional Black-Scholes equation with risk free rate $r$. Here $r>0$ is risk free interest rate, $S_{i}$ the $i$-th underlying asset with dividend rate $q_{i}(i=1, \cdots, n)$, $A=\left[a_{i j}\right]_{i, j=0}^{n}$ a non-negative definite $(n+1) \times(n+1)$ matrix and $V\left(S_{0}, S_{1}, \cdots, S_{n}, t\right)$ the price of an option derived from underlying assets $S_{0}, S_{1}, \cdots, S_{n}$ at time $t$. The existence and representation of the solution to equation (1) are described in [3. The following theorem is our main result.

Theorem 1 Under the transformation

$$
z_{1}=S_{0}^{\alpha_{0}} S_{1}^{\alpha_{1}}, \quad z_{i}=S_{i}, \quad i=2, \cdots, n,
$$

equation (1) is transformed into an $n$-dimensional Black-Scholes equation with risk free rate $r$. In other words, the equation (11) has a solution of the form

$$
V\left(S_{0}, S_{1}, \cdots, S_{n}, t\right)=U\left(z_{1}, \cdots, z_{n}, t\right) .
$$

where $U\left(z_{1}, \cdots, z_{n}, t\right)$ is a solution to the following $n$ dimensional Black-Scholes equation with risk free rate $r$ :

$$
\frac{\partial U}{\partial t}+\frac{1}{2} \sum_{i, j=1}^{n} \bar{a}_{i j} z_{i} z_{j} \frac{\partial^{2} U}{\partial z_{i} \partial z_{j}}+\sum_{i=1}^{n}\left(r-\bar{q}_{i}\right) z_{i} \frac{\partial U}{\partial z_{i}}-r U=0
$$


Here $\bar{a}_{i j}, \bar{q}_{i}$ are provided as follows:

$$
\begin{aligned}
& \bar{a}_{i j}= \begin{cases}a_{00} \alpha_{0}^{2}+a_{01} \alpha_{0} \alpha_{1}+a_{10} \alpha_{1} \alpha_{0}+a_{11} \alpha_{1}^{2}, & i=j=1, \\
a_{0 j} \alpha_{0}+a_{1 j} \alpha_{1}, & i=1, j=2, \cdots, n, \\
a_{i 0} \alpha_{0}+a_{i 1} \alpha_{1}, & i=2, \cdots, n, j=1, \\
a_{i j}, & i, j=2, \cdots, n,\end{cases} \\
& \bar{q}_{k}= \begin{cases}r-\sum_{i=0}^{1}\left(r-q_{i}-\frac{1}{2} a_{i i}\right) \alpha_{i}-\frac{1}{2} \sum_{i, j=0}^{1} a_{i j} \alpha_{i} \alpha_{j}, & k=1, \\
q_{k}, & k=2, \cdots, n .\end{cases}
\end{aligned}
$$

Proof If we rewrite the derivatives of $V$ on $S_{i}(i=0, \cdots, n)$ in (1) by the derivatives of $U$ on $z_{i}(i=1, \cdots, n)$ using (3), then we have

$$
\begin{aligned}
& \frac{\partial V}{\partial S_{0}} S_{0}=\alpha_{0} \frac{\partial U}{\partial z_{1}} z_{1}, \quad \frac{\partial V}{\partial S_{1}} S_{1}=\alpha_{1} \frac{\partial U}{\partial z_{1}} z_{1}, \quad \frac{\partial V}{\partial S_{i}} S_{i}=\frac{\partial U}{\partial z_{i}} z_{i}, \quad i=2, \cdots, n, \\
& \frac{\partial^{2} V}{\partial S_{0}^{2}} S_{0}^{2}=\alpha_{0}^{2} \frac{\partial^{2} U}{\partial z_{1}^{2}} z_{1}^{2}+\alpha_{0}\left(\alpha_{0}-1\right) \frac{\partial U}{\partial z_{1}} z_{1}, \\
& \frac{\partial^{2} V}{\partial S_{1}^{2}} S_{1}^{2}=\alpha_{1}^{2} \frac{\partial^{2} U}{\partial z_{1}^{2}} z_{1}^{2}+\alpha_{1}\left(\alpha_{1}-1\right) \frac{\partial U}{\partial z_{1}} z_{1}, \\
& \frac{\partial^{2} V}{\partial S_{0} \partial S_{1}} S_{0} S_{1}=\alpha_{0} \alpha_{1} \frac{\partial^{2} U}{\partial z_{1}^{2}} z_{1}^{2}+\alpha_{0} \alpha_{1} \frac{\partial U}{\partial z_{1}} z_{1}, \\
& \frac{\partial^{2} V}{\partial S_{0} \partial S_{j}} S_{0} S_{j}=\alpha_{0} \frac{\partial^{2} U}{\partial z_{1} \partial z_{j}} z_{1} z_{j}, \quad j=2, \cdots, n \\
& \frac{\partial^{2} V}{\partial S_{1} \partial S_{j}} S_{1} S_{j}=\alpha_{1} \frac{\partial^{2} U}{\partial z_{1} \partial z_{j}} z_{1} z_{j}, \quad j=2, \cdots, n \\
& \frac{\partial^{2} V}{\partial S_{i} \partial S_{j}} S_{i} S_{j}=\frac{\partial^{2} U}{\partial z_{i} \partial z_{j}} z_{i} z_{j}, \quad i, j=2, \cdots, n .
\end{aligned}
$$

If we expand the terms of second order derivatives in (1) as follows

$$
\sum_{i, j=0}^{n} b_{i j}=\sum_{i, j=0}^{1} b_{i j}+\sum_{i=0}^{1} \sum_{j=2}^{n} b_{i j}+\sum_{i=2}^{n} \sum_{j=0}^{1} b_{i j}+\sum_{i, j=2}^{n} b_{i j}
$$

and substitute the above derivatives into here, then we can easily have (44) and (5). Then the $n$-dimensional matrix $\bar{A}=\left[\bar{a}_{i j}\right]_{i, j=1}^{n}$ is evidently symmetric and non-negative. In fact, 
for any $\boldsymbol{\xi}=\left(\xi_{1}, \cdots, \xi_{n}\right)^{\perp} \in \mathbf{R}^{n}$ (the superscript " $\perp$ " denotes transpose), we have

$$
\begin{aligned}
\boldsymbol{\xi}^{\perp} \overline{\mathbf{A}} \boldsymbol{\xi} & =\sum_{i, j=1}^{n} \bar{a}_{i j} \xi_{i} \xi_{j} \\
& =\bar{a}_{11} \xi_{1}^{2}+\sum_{j=2}^{n} \bar{a}_{1 j} \xi_{1} \xi_{j}+\sum_{i=2}^{n} \bar{a}_{i 1} \xi_{i} \xi_{1}+\sum_{i, j=2}^{n} \bar{a}_{i j} \xi_{i} \xi_{j} \\
& =\sum_{i, j=0}^{1} a_{i j} \alpha_{i} \alpha_{j} \xi_{1}^{2}+\sum_{j=2}^{n}\left(\sum_{i=0}^{1} a_{i j} \alpha_{i}\right) \xi_{1} \xi_{j}+\sum_{i=2}^{n}\left(\sum_{j=0}^{1} a_{i j} \alpha_{j}\right) \xi_{i} \xi_{1}+\sum_{i, j=2}^{n} a_{i j} \xi_{i} \xi_{j} \\
& =\sum_{i, j=0}^{1} a_{i j}\left(\alpha_{i} \xi_{1}\right)\left(\alpha_{j} \xi_{1}\right)+\sum_{i=0}^{n} \sum_{j=2}^{n} a_{i j}\left(\alpha_{i} \xi_{1}\right) \xi_{j}+\sum_{i=2}^{n} \sum_{j=0}^{1} a_{i j} \xi_{i}\left(\alpha_{j} \xi_{1}\right)+\sum_{i, j=2} a_{i j} \xi_{i} \xi_{j}
\end{aligned}
$$

So if we let $\boldsymbol{\eta}=\left(\alpha_{0} \xi_{1}, \alpha_{1} \xi_{1}, \xi_{2}, \cdots, \xi_{n}\right)^{\perp} \in \mathbf{R}^{n+1}$, then from the nonnegativeness of $A$, we have

$$
\boldsymbol{\xi}^{\perp} \bar{A} \boldsymbol{\xi}=\boldsymbol{\eta}^{\perp} A \boldsymbol{\eta} \geq 0
$$

which completes the proof of theorem 1. (QED) 1 .

If we apply the transformation (2) repeatedly, then we can get the following corollary

Corollary 1 Let $k \in\{1,2, \cdots, n\}$. Under the transformation

$$
z_{k}=S_{0}^{\alpha_{0}} \cdots S_{k}^{\alpha_{k}}, \quad z_{i}=S_{i}, \quad i=k+1, \cdots, n .
$$

the equation (1) is transformed into an $(n+1-k)$-dimensional Black-Scholes equation.

Let consider a terminal condition

$$
V\left(S_{0}, S_{1}, \cdots, S_{n}, T\right)=P\left(S_{0}, S_{1}, \cdots, S_{n}\right)
$$

of $(n+1)$-dimensional Black-Scholes equation (11). Then from theorem 1, we have the following corollary 2 .

Corollary 2 Assume that there exists an $n$-dimensional function $F$ such that

$$
P\left(S_{0}, S_{1}, S_{2}, \cdots, S_{n}\right)=F\left(S_{0}^{\alpha_{0}}, S_{1}^{\alpha_{1}}, S_{2}, \cdots, S_{n}\right)
$$

Then by the change of variables given by (2) and (3), the terminal value problem given by (1) and (7) is transformed into the $n$-dimensional Black-Scholes equation's terminal value problem given by (4) and

$$
U\left(z_{1}, \cdots, z_{n}, T\right)=F\left(z_{1}, \cdots, z_{n}\right) .
$$

Remark 1 As shown in [4], if the expiry payoff function $P\left(S_{0}, S_{1}, \cdots, S_{n}\right)$ has the homogeneity for its variables:

$$
P\left(a S_{0}, a S_{1}, \cdots, a S_{n}\right)=a P\left(S_{0}, S_{1}, \cdots, S_{n}\right), \forall a>0
$$


then by the change of numeraire

$$
U=\frac{V}{S_{0}}, \quad z_{i}=\frac{S_{i}}{S_{0}}, \quad i=1, \cdots, n,
$$

the $(n+1)$-dimensional terminal value problem given by (10) and (7) is transformed into a terminal value problem for an $n$-dimensional Black-Scholes equation with risk free rate 0 . The new transformed expiry payoff function $F$ is given by

$$
F\left(z_{1}, \cdots, z_{n}\right) \triangleq P\left(1, z_{1}, \cdots, z_{n}\right) .
$$

Then the new expiry payoff function $F$ no more has the homogeneity for its variables. For example

$$
P\left(S_{0}, S_{1}, S_{2}\right)=\max \left(S_{0}, S_{1}, S_{2}\right)
$$

has the homogeneity but its new transformed 2 dimensional function

$$
F\left(z_{1}, z_{2}\right)=\max \left(1, z_{1}, z_{2}\right)
$$

has no homogeneity. Thus the change of numeraire only can reduce the number of sources of risks by one and it is impossible to use repeatedly.

Remark 2 In corollary 2, if the new expiry payoff $F$ has the same property with $P$ in (8), then we can use the transformation (2) repeatedly. For example, if

$$
P\left(S_{0}, S_{1}, S_{2}\right)=\max \left(S_{0} S_{1} S_{2}-K, 0\right)
$$

and we apply the transformation $z=S_{0} \cdot S_{1}$, then the new expiry payoff $F$ is also given by

$$
F\left(z_{1}, z_{2}\right)=\max \left(z_{1} z_{2}-K, 0\right),
$$

thus it is also the function of $z_{1} \cdot z_{2}$ and we can use the transformation (2) once more, and then we have one-dimensional function $G(x)=\max (x-K, 0)$.

Remark 3 In our opinion, in multi-dimensional Black-Scholes equations, the principle to reduce dimension is based on its invariance under the transformation (6) and the change of numeraire (10). Thus in pricing financial derivatives problems derived to multi-dimensional Black-Scholes equations, the possibility to reduce dimension depends on the structures of their expiry payoff functions. If the price model of a derivative can be derived to BlackScholes equation and its expiry payoff is a function of some group of risk source variables such as (6) and furthermore, if the new expiry payoff function of group variables has homogeneity, then using (6) and (10) we can reduce the dimension of the problem by two or more.

\section{$3 \quad$ Examples}

\subsection{Options with Foreign Currency Strike Price}

This example is studied in [1] by expectation method and in 4] by PDE method. For details about the financial background, we refer to [1,4. Here we only mention the use of the transformation given by the equation (2). 
Problem: The underlying stock is traded in UK pounds and the option exercise price is in US dollars. At $t=0$ the option is an at-the-money option (that is, the strike price is the same with the underlying stock price $3,5,5]$ ) when the strike price is expressed in UK pounds. This pound strike price is converted into dollars at $t=0$. The dollar strike price computed like this is kept constant during the life of the option. At the expiry date $t=T$, the option holder can pay the fixed dollar strike price to buy the underlying stock. Find the fair price of this option.

\section{Mathematical model}

Let denote by $S(t)$ the stock price (in UK pounds), $r_{p}$ the short rate in UK pound market, $r_{d}$ the short rate in US dollar, $X(t)$ dollar/pound exchange rate (then $Y(t)=X(t)^{-1}$ is pound /dollar exchange rate), $K_{d}$ the strike price expressed in US dollar and $K_{p}(t)$ the strike price expressed in UK pound.

Assumption 1) The stock price $S(t)$ satisfies geometric Brown motion:

$$
d S(t)=\alpha_{S} S(t) d t+\sigma_{S} S(t) d W^{S}(t) \text { (objective measure). }
$$

2) $r_{p}$ and $r_{d}$ are deterministic constants.

3) Dollar/pound exchange rate $X(t)$ satisfies Garman-Kohlhagen model 2]:

$$
d X(t)=\alpha_{X} X(t) d t+\sigma_{X} X(t) d W^{X}(t) \text { (objective measure). }
$$

Here $W^{S}(t)$ and $W^{X}(t)$ are the scalar Wiener processes and have the following relations:

$$
d W^{S}(t) \cdot d W^{X}(t)=\rho d t, \quad|\rho|<1 .
$$

Explanation about strike price: $K_{p}(0)=S(0), K_{d}=K_{p}(0) \cdot X(0)=S(0) \cdot X(0) \equiv$ constant. The dollar strike price is constant but the pound strike price randomly varies as a result of varying exchange rate:

$$
K_{p}(t)=K_{d} \cdot X(t)^{-1}=S(0) \cdot X(0) \cdot X(t)^{-1} .
$$

Explanation about maturity payoff: The US dollar price of the option at maturity is

$$
F_{d}=\max \left(S(T) \cdot X(T)-K_{d}, 0\right) .
$$

Then the UK pound price of the option at maturity is

$$
\begin{aligned}
F_{p} & =\max \left(S(T)-K_{p}(T), 0\right) \\
& =\max \left(S(T)-S(0) \cdot Y(0)^{-1} \cdot Y(T), 0\right) .
\end{aligned}
$$

Now we derive the PDE model for pricing the option in dollars. Let $V_{d}=V(S, X, t)$ be the price of the option in US dollars. By $\Delta$-hedging, construct a portfolio $\Pi$ as

$$
\Pi=V-\Delta_{1} S X-\Delta_{2} X .
$$

(the dollar price of this portfolio consists of an option, $\Delta_{1}$ shares of stocks and $\Delta_{2}$ UK pounds.) Choose $\Delta_{1}, \Delta_{2}$ such that $\Pi$ is risk-free in $(t, t+d t)$, i.e.

$$
d \Pi=r_{d} \Pi d t .
$$


Then we easily can derive the following PDE pricing model [4]:

$$
\begin{aligned}
& \frac{\partial V}{\partial t}+\frac{1}{2}\left[\sigma_{S}^{2} S^{2} \frac{\partial^{2} V}{\partial S^{2}}+2 \rho \sigma_{S} \sigma_{X} S X \frac{\partial^{2} V}{\partial S \partial X}+\sigma_{X}^{2} X^{2} \frac{\partial^{2} V}{\partial X^{2}}\right] \\
& +\left(r_{p}-\rho \sigma_{S} \sigma_{X}\right) S \frac{\partial V}{\partial S}+\left(r_{d}-r_{p}\right) X \frac{\partial V}{\partial X}-r_{d} V=0 \\
& V(S, X, T)=\max \left(S \cdot X-K_{d}, 0\right) .
\end{aligned}
$$

Here we remind that $S$ is the variable representing stock price, $X$ the variable representing dollar/pound exchange rate, $T$ the maturity and $V$ the option price.

The equation (11) is a two-dimensional Black-Scholes equation and $T$-payoff function (12) does not satisfy homogeneity, but it is a function of the group variable

$$
z=S X \text {. }
$$

(This change of variable transforms the stock price expressed in UK pound into UD dollar price.) Thus from corollary 2 of our theorem 1, by the transformation (13) our problem is transformed into the following one dimensional problem:

$$
\begin{aligned}
& \frac{\partial V}{\partial t}+\frac{1}{2}\left(\sigma_{S}^{2}+2 \rho \sigma_{S} \sigma_{X}+\sigma_{X}^{2}\right) z^{2} \frac{\partial^{2} V}{\partial z^{2}}+r_{d} z \frac{\partial V}{\partial z}-r_{d} V=0, \\
& V(z, T)=\max \left(z-K_{d}, 0\right) .
\end{aligned}
$$

The problem (14) can be seen as an ordinary UK dollar call option pricing problem. By the standard Black-Scholes formula,

$$
V(z, t)=z N\left(d_{1}\right)-K_{d} e^{-r_{d}(T-t)} N\left(d_{2}\right)
$$

where

$$
\begin{aligned}
& d_{1}=\frac{\ln \frac{z}{K_{d}}+\left(r_{d}+\frac{1}{2} \sigma_{S, X}^{2}\right)(T-t)}{\sigma_{S, X} \sqrt{T-t}}, \quad d_{2}=d_{1}-\sigma_{S, X} \sqrt{T-t}, \\
& \sigma_{S, X}^{2}=\sigma_{S}^{2}+2 \rho \sigma_{S} \sigma_{X}+\sigma_{X}^{2} .
\end{aligned}
$$

If we return to the original variables $(S, X)$, then we have the dollar price of the option:

$$
V_{d}(S, X, t)=S X N\left(d_{1}\right)-K_{d} e^{-r_{d}(T-t)} N\left(d_{2}\right),
$$

where

$$
d_{1}=\frac{\ln \frac{S X}{K_{d}}+\left(r_{d}+\frac{1}{2} \sigma_{S, X}^{2}\right)(T-t)}{\sigma_{S, X} \sqrt{T-t}}, \quad d_{2}=d_{1}-\sigma_{S, X} \sqrt{T-t} .
$$

Considering $V_{p}=V_{d} \cdot X^{-1}$ and $K_{d}=S(0) \cdot X(0)$, we have the pound price of option:

$$
V_{p}(S, X, t)=S N\left(d_{1}\right)-\frac{S_{0} X_{0}}{X} e^{-r_{d}(T-t)} N\left(d_{2}\right),
$$

where

$$
d_{1}=\frac{\ln \frac{S X}{S_{0} X_{0}}+\left(r_{d}+\frac{1}{2} \sigma_{S, X}^{2}\right)(T-t)}{\sigma_{S, X} \sqrt{T-t}}, \quad d_{2}=d_{1}-\sigma_{S, X} \sqrt{T-t}
$$




\subsection{Basket Option with the Expiry Payoff of the Geometric Mean}

Assume that the prices $m$ underlying assets $S_{0}, \cdots, S_{m-1}$ follow geometric Brownian motions and the expiry payoff of an option is

$$
V\left(S_{0}, \cdots, S_{m-1}, T\right)=\left(S_{0}^{\alpha_{0}}, \cdots, S_{m-1}^{\alpha_{m-1}}-K\right)^{+}
$$

where $\sum \alpha_{i}=1, \alpha_{i} \geq 0$. Such an option is called a basket option with the expiry payoff of the geometric mean of $m$ underlying assets $[3$.

The mathematical model for this option is the terminal value problem (11) and (15) of $m$-dimensional Black-Scholes equation when $n+1=m$.

Under the transformation

$$
z=S_{0}^{\alpha_{0}} \cdots S_{m-1}^{\alpha_{m-1}}
$$

we have

$$
\begin{aligned}
& S_{i} \frac{\partial V}{\partial S_{i}}=\alpha_{i} z \frac{\partial V}{\partial z}, \quad i=0, \cdots, m-1, \\
& S_{i}^{2} \frac{\partial^{2} V}{\partial S_{i}^{2}}=\alpha_{i}^{2} z^{2} \frac{\partial^{2} V}{\partial z^{2}}+\alpha_{i}^{2} z \frac{\partial V}{\partial z}-\alpha_{i} z \frac{\partial V}{\partial z}, \\
& S_{i} S_{j} \frac{\partial^{2} V}{\partial S_{i} \partial S_{j}}=\alpha_{i} \alpha_{j} z^{2} \frac{\partial^{2} V}{\partial z^{2}}+\alpha_{i} \alpha_{j} z \frac{\partial V}{\partial z}, \quad(i \neq j) .
\end{aligned}
$$

If we substitute the above derivative expressions into the equation (1) when $n+1=m$ and consider $\sum \alpha_{i}=1$, then we have

$$
\begin{aligned}
\frac{\partial V}{\partial t} & +\frac{1}{2}\left[\sum_{i, j=0}^{m-1} a_{i j} \alpha_{i} \alpha_{j} z^{2} \frac{\partial^{2} V}{\partial z^{2}}+\left(\sum_{i, j=0}^{m-1} a_{i j} \alpha_{i} \alpha_{j}-\sum_{i=0}^{m-1} a_{i i} \alpha_{i}\right) z \frac{\partial V}{\partial z}\right] \\
& +\left(r-\sum_{i=0}^{m-1} q_{i} \alpha_{i}\right) z \frac{\partial V}{\partial z}-r V=0
\end{aligned}
$$

Let denote

$$
\hat{\sigma}^{2}:=\sum_{i, j=0}^{m-1} a_{i j} \alpha_{i} \alpha_{j}, \quad \hat{q}:=\sum_{i=0}^{m-1}\left(q_{i}+\frac{1}{2} a_{i i}\right) \alpha_{i}-\frac{1}{2} \hat{\sigma}^{2} .
$$

Then we have the following terminal value problem of one dimensional Black-Scholes equation (standard call option):

$$
\begin{aligned}
& \frac{\partial V}{\partial t}+\frac{1}{2} \hat{\sigma}^{2} z^{2} \frac{\partial^{2} V}{\partial z^{2}}+(r-\hat{q}) z \frac{\partial V}{\partial z}-r V=0, \\
& V(z, T)=(z-K)^{+} .
\end{aligned}
$$

If we use the standard Black-Scholes formula and return to the original variables, then we have the pricing formula of a basket option with the expiry payoff of the geometric mean:

$$
V\left(S_{0}, \cdots, S_{m-1}, t\right)=e^{-\hat{q}(T-t)} S_{0}^{\alpha_{0}} \cdots S_{m-1}^{\alpha_{m-1}} N\left(d_{1}\right)-K e^{-r(T-t)} N\left(d_{2}\right),
$$


where

$$
d_{1}=\frac{\ln \frac{S_{0}^{\alpha_{0} \ldots \cdot S_{m-1}^{\alpha_{m-1}}}}{K}+\left(r-\hat{q}+\frac{1}{2} \hat{\sigma}^{2}\right)(T-t)}{\hat{\sigma} \sqrt{T-t}}, \quad d_{2}=d_{1}-\hat{\sigma} \sqrt{T-t} .
$$

\subsection{Pricing a European Call Foreign Currency Option}

This problem was studied in [6] under a general condition with stochastic short rates and stochastic exchange rates. They consider the option as a interest rate derivative, so that the partial differential equations of the prices that they derived are not standard multidimensional Black-Scholes equations. Thus our result can not be directly applied to their equation. Fortunately, as mentioned in [4, the prices of zero coupon bonds under the Vasicek model, Ho-Lee model or Hull-White model follow geometric Brown motions and the corresponding short rate is a deterministic function of the price of zero coupon bond. So here we consider the option as a zero coupon bond derivative, just as in [4], and then we apply our theorem.

Problem: A holder of an European call foreign currency option has a right to fix the exchange rate as a strike exchange rate. Find the fair price of this option.

We denote by $r_{1}(t)$ the short rate in domestic currency, $r_{2}(t)$ the short rate in foreign currency, $F(t)$ domestic currency / foreign currency exchange rate, $K$ the strike exchange rate (domestic / foreign), and $T$ the expiry date. Under this notation, the expiry pay off of our European call foreign currency option is given by

$$
[F(T)-K]^{+} .
$$

Assumptions: All discussion is done under the risk neutral measure $Q$ (domestic martingale measure). In what follows, $a_{1}, a_{2}, b_{1}, b_{2}$ are all positive constants, $\sigma_{1}, \sigma_{2}, \sigma_{3}$ are linear independent constant vectors and $\left\{W_{t} ; 0 \leq t \leq T\right\}=\left\{\left(W_{t}^{1}, W_{t}^{2}, W_{t}^{3}\right) ; 0 \leq t \leq T\right\}$ is a standard 3 dimensional Wiener process satisfying the following conditions:

$$
E\left(d W_{t}^{i}\right)=0, \quad \operatorname{Var}\left(d W_{t}^{i}\right)=d t, \quad \operatorname{Cov}\left(d W_{t}^{i}, d W_{t}^{j}\right)=0(i \neq j), 1 \leq i, j \leq 3 .
$$

1) The domestic and foreign short rates follow Vasicek model:

$$
d r_{i}=\left(b_{i}-a_{i} r_{i}\right) d t+\sigma_{i} \cdot d W(t), \quad i=1,2 .
$$

2) The exchange rate $F(t)$ follows Garman-Kohlhagen model [2]:

$$
d F(t)=F(t)\left(r_{1}(t)-r_{2}(t)\right) d t+F(t) \sigma_{3} \cdot d W(t) .
$$

3) The price $V$ of the option in domestic currency is given as a deterministic function $V=C\left(r_{1}, r_{2}, F, t\right)$ of domestic short rate, foreign short rate and exchange rate and assume

$$
V \in C^{2,1}(D \times[0, T)), \quad D=(-\infty, \infty) \times(-\infty, \infty) \times(0, \infty) .
$$

Dynamics of the price of zero coupon bond: Let denote the price of domestic zero coupon bond with maturity $T$ by $p_{1}\left(t, r_{1} ; T\right)$ (in domestic currency) and denote the price 
of foreign zero coupon bond with maturity $T$ by $p_{2}\left(t, r_{2} ; T\right)$ (in foreign currency). Then the price of zero coupon bond $p_{i}\left(t, r_{i} ; T\right)$ satisfies the following equation [5]:

$$
\begin{aligned}
& \frac{\partial p_{i}}{\partial t}+\frac{1}{2}\left|\sigma_{i}\right|^{2} \frac{\partial^{2} p_{i}}{\partial r_{i}^{2}}+\left(b_{i}-a_{i} r_{i}-\lambda_{i}\left|\sigma_{i}\right|\right) \frac{\partial p_{i}}{\partial r_{i}}-r_{i} p_{i}=0, \\
& p_{i}\left(T, r_{i} ; T\right)=1 .
\end{aligned}
$$

Here $\lambda_{1}=0$ is the price of domestic market risk (under the domestic martingale measure) and $\lambda_{2} \neq 0$ is the price of foreign market risk (under the domestic martingale measure), $|\sigma|$ denotes the length of vector $\sigma$. And its solution is expressed by

$$
p_{i}\left(t, r_{i} ; T\right)=A_{i}(t, T) e^{-B_{i}(t, T) r_{i}}, \frac{\partial p_{i}}{\partial r_{i}}=-B_{i}(t, T) p_{i}, B_{i}(t, T)=\frac{1}{a_{i}}\left(1-e^{-a_{i}(T-t)}\right),
$$

and so the short rate $r_{i}(t)$ is a deterministic function of $p_{i}=p_{i}\left(t, r_{i}, T\right)$ :

$$
r_{i}=r_{i}\left(t, p_{i}\right)=-\frac{1}{B_{i}(t)}\left(\ln p_{i}-A_{i}(t)\right)=-\frac{1}{B_{i}(t)} \ln p_{i}+\frac{A_{i}(t)}{B_{i}(t)}
$$

As shown in [4, the dynamics of the zero coupon bond price follows geometric Brown motion: In fact

$$
\begin{aligned}
d p_{i} & =\left(\frac{\partial p_{i}}{\partial t}+\frac{1}{2}\left|\sigma_{i}\right|^{2} \frac{\partial^{2} p_{i}}{\partial r_{i}^{2}}\right) d t+\frac{\partial p_{i}}{\partial r_{i}} d r_{i} \\
& =\left(\frac{\partial p_{i}}{\partial t}+\frac{1}{2}\left|\sigma_{i}\right|^{2} \frac{\partial^{2} p_{i}}{\partial r_{i}^{2}}+\left(b_{i}-a_{i} r_{i}\right) \frac{\partial p_{i}}{\partial r_{i}}\right) d t+\frac{\partial p_{i}}{\partial r_{i}} \sigma_{i} \cdot d W(t) \\
& =\left(r p_{i}+\lambda_{i}\left|\sigma_{i}\right| \frac{\partial p_{i}}{\partial r_{i}}\right) d t+\frac{\partial p_{i}}{\partial r_{i}} \sigma_{i} \cdot d W(t) \\
& =\left(r_{i}-\lambda_{i}\left|\sigma_{i}\right| B_{i}(t)\right) p_{i} d t+p_{i} B_{i}(t) \sigma_{i} \cdot d W(t)
\end{aligned}
$$

Thus we get

$$
\begin{aligned}
& d p_{i}=\alpha_{i}(t) p_{i} d t+p_{i} \Sigma_{i}(t) \cdot d W(t), \quad i=1,2, \\
& \Sigma_{i}(t)=-B_{i}(t) \sigma_{i} .
\end{aligned}
$$

PDE Model and Solving: Now we can attack the pricing problem. Denote by

$$
p_{i}=p_{i}(t ; T) .
$$

Since $p_{i}(T, T)=1$, then the price of our option can be rewritten as

$$
V_{T}=\max \left(F p_{2}-K p_{1}, 0\right)
$$

From the assumption 3) and the fact that $r_{1}(t)=r_{1}\left(t, p_{1}\right)$ and $r_{2}(t)=r_{2}\left(t, p_{2}\right)$, the domestic price of the option at time $t$ can be rewritten as a function

$$
V=V\left(p_{1}, p_{2}, F, t\right)
$$

of the zero coupon bond prices. By $\Delta$-hedging, construct a portfolio $\Pi$ as

$$
\Pi=V-\Delta_{1} p_{1}-\Delta_{2} p_{2} F-\Delta_{3} F . \quad \text { (in domestic currency) }
$$


This portfolio consists of an option, $\Delta_{1}$ shares of domestic zero coupon bond, $\Delta_{2}$ shares of foreign zero coupon bond and $\Delta_{3}$ units of foreign currency. Choose $\Delta_{1}, \Delta_{2}, \Delta_{3}$ such that $\Pi$ is risk-free in $(t, t+d t)$, i.e. $d \Pi=r_{1} \Pi d t$. This is equivalent to the following equality

$$
d V-\Delta_{1} d p_{1}-\Delta_{2} d\left(p_{2} F\right)-\Delta_{3} d F-\Delta_{3} r_{2} d t F=r_{1}\left(t, p_{1}\right)\left(V-\Delta_{1} p_{1}-\Delta_{2} p_{2} F-\Delta_{3} F\right) d t .
$$

By the three dimensional Itô formula, we have

$$
\begin{aligned}
d V= & \frac{\partial V}{\partial p_{1}} d p_{1}+\frac{\partial V}{\partial p_{2}} d p_{2}+\frac{\partial V}{\partial F} d F+\left\{\frac{\partial V}{\partial t}+\frac{1}{2}\left[\left|\Sigma_{1}\right|^{2} p_{1}^{2} \frac{\partial^{2} V}{\partial p_{1}^{2}}\right.\right. \\
& +\left|\Sigma_{2}\right|^{2} p_{2}^{2} \frac{\partial^{2} V}{\partial p_{2}^{2}}+\left|\sigma_{3}\right|^{2} F^{2} \frac{\partial^{2} V}{\partial F^{2}}+2 \Sigma_{1} \cdot \Sigma_{2} p_{1} p_{2} \frac{\partial^{2} V}{\partial p_{1} \partial p_{2}} \\
& \left.\left.+2 \Sigma_{1} \cdot \sigma_{3} p_{1} F \frac{\partial^{2} V}{\partial p_{1} \partial F}+2 \Sigma_{2} \cdot \sigma_{3} p_{2} F \frac{\partial^{2} V}{\partial p_{2} \partial F}\right]\right\} d t \\
d\left(p_{2} F\right) & =p_{2} d F+F d p_{2}+\Sigma_{2} \cdot \sigma_{3} p_{2} F d t .
\end{aligned}
$$

If we substitute above two expressions into (20), then we get

$$
\begin{aligned}
\left(\frac{\partial V}{\partial p_{1}}-\right. & \left.\Delta_{1}\right) d p_{1}+\left(\frac{\partial V}{\partial p_{2}}-\Delta_{2} F\right) d p_{2}+\left(\frac{\partial V}{\partial F}-\Delta_{2} p_{2}-\Delta_{3}\right) d F \\
& +\left\{\frac{\partial V}{\partial t}+\frac{1}{2}\left[\left|\Sigma_{1}\right|^{2} p_{1}^{2} \frac{\partial^{2} V}{\partial p_{1}^{2}}+\left|\Sigma_{2}\right|^{2} p_{2}^{2} \frac{\partial^{2} V}{\partial p_{2}^{2}}+\left|\sigma_{3}\right|^{2} F^{2} \frac{\partial^{2} V}{\partial F^{2}}\right.\right. \\
& \left.\left.+2 \Sigma_{1} \cdot \Sigma_{2} p_{1} p_{2} \frac{\partial^{2} V}{\partial p_{1} \partial p_{2}}+2 \Sigma_{1} \cdot \sigma_{3} p_{1} F \frac{\partial^{2} V}{\partial p_{1} \partial F}+2 \Sigma_{2} \cdot \sigma_{3} p_{2} F \frac{\partial^{2} V}{\partial p_{2} \partial F}\right]\right\} d t \\
& -\Delta_{2} \Sigma_{2} \cdot \sigma_{3} p_{2} F d t-\Delta_{3} r_{2}\left(t, p_{2}\right) d t \cdot F \\
= & r_{1}\left(t, p_{1}\right)\left(V-\Delta_{1} p_{1}-\Delta_{2} p_{2} F-\Delta_{3} F\right) d t .
\end{aligned}
$$

Here we choose $\Delta_{1}$ and $\Delta_{2}$ such that

$$
\frac{\partial V}{\partial p_{1}}-\Delta_{1}=0, \quad \frac{\partial V}{\partial p_{2}}-\Delta_{2} F=0, \quad \frac{\partial V}{\partial F}-\Delta_{2} p_{2}-\Delta_{3}=0,
$$

equivalently,

$$
\Delta_{1}=\frac{\partial V}{\partial p_{1}}, \quad \Delta_{2}=\frac{1}{F} \frac{\partial V}{\partial p_{2}}, \quad \Delta_{3}=\frac{\partial V}{\partial F}-\frac{p_{2}}{F} \frac{\partial V}{\partial p_{2}} .
$$

Then we have

$$
\begin{aligned}
& \frac{\partial V}{\partial t}+r_{1}\left(t, p_{1}\right) \frac{\partial V}{\partial p_{1}} p_{1}+\left[r_{2}\left(t, p_{2}\right)-\Sigma_{2}(t) \cdot \sigma_{3}\right] \frac{\partial V}{\partial p_{2}} p_{2} \\
& \quad+\left[r_{1}\left(t, p_{1}\right)-r_{2}\left(t, p_{2}\right)\right] F \frac{\partial V}{\partial F}+\frac{1}{2}\left[\left|\Sigma_{1}(t)\right|^{2} p_{1}^{2} \frac{\partial^{2} V}{\partial p_{1}^{2}}\right. \\
& \quad+\left|\Sigma_{2}(t)\right|^{2} p_{2}^{2} \frac{\partial^{2} V}{\partial p_{2}^{2}}+\left|\sigma_{3}\right|^{2} F^{2} \frac{\partial^{2} V}{\partial F^{2}}+2 \Sigma_{1}(t) \cdot \Sigma_{2}(t) p_{1} p_{2} \frac{\partial^{2} V}{\partial p_{1} \partial p_{2}} \\
& \left.\quad+2 \Sigma_{1}(t) \cdot \sigma_{3} p_{1} F \frac{\partial^{2} V}{\partial p_{1} \partial F}+2 \Sigma_{2}(t) \cdot \sigma_{3} p_{2} F \frac{\partial^{2} V}{\partial p_{2} \partial F}\right]-r_{1}\left(t, p_{1}\right) V=0 .
\end{aligned}
$$


The problem (21) and (19) is the pricing model for European call foreign currency option which is considered as a derivative of two countrys zero coupon bonds.

The equation (21) has a simillar form of Black-Scholes equation but the terms of first order derivatives and itself of unknown function have strongly varying coefficients which depend on space variables. Although our theorem deals with constant coefficient BlackScholes equation, but the change of variables

$$
z=p_{2} \cdot F
$$

does work well. This change of variables composes the price of foreign zero coupon bond and the exchange rate to the domestic price of foreign zero coupon bond. By this change of variables, the space 3 -dimensional problem given by (21) and (19) is transformed into the following space 2-dimensional problem:

$$
\begin{aligned}
\frac{\partial V}{\partial t}+ & r_{1}\left(t, p_{1}\right) \frac{\partial V}{\partial p_{1}} p_{1}+r_{1}\left(t, p_{1}\right) \frac{\partial V}{\partial z} z+\frac{1}{2}\left[\left|\Sigma_{1}(t)\right|^{2} p_{1}^{2} \frac{\partial^{2} V}{\partial p_{1}^{2}}+\left|\Sigma_{2}(t)+\sigma_{3}\right|^{2} z^{2}\right. \\
& \left.\frac{\partial^{2} V}{\partial z^{2}}+2 \Sigma_{1}(t) \cdot\left(\Sigma_{2}(t)+\sigma_{3}\right) p_{1} z \frac{\partial^{2} V}{\partial p_{1} \partial z}\right]-r_{1}\left(t, p_{1}\right) V=0 \\
V_{T}= & \max \left(z-K p_{1}, 0\right) .
\end{aligned}
$$

The original expiry payoff function (19) has no homogeneity on its variables $\left(p_{1}, p_{2}, F\right)$ but the changed expiry payoff function of the problem (22) has homogeneity on its new variables $\left(z, p_{1}\right)$, and thus by theorem 1 of [4] we can use the standard change of numeraire

$$
U=\frac{V}{p_{1}}, \quad y=\frac{z}{p_{1}}\left(=\frac{p_{2} F}{p_{1}}\right) .
$$

This change of variables transforms the bond price and domestic price of foreign zero coupon bond into relative price with respect to the zero coupon bond price and we have the following terminal value problem of 1-dimensional Black-Scholes equation with risk free rate 0:

$$
\begin{aligned}
& \frac{\partial U}{\partial t}+\frac{1}{2}\left|\Sigma_{1}(t)-\Sigma_{2}(t)-\sigma_{3}\right|^{2} \frac{\partial^{2} U}{\partial y^{2}} y^{2}=0, \\
& U(y, T)=\max (y-K, 0) .
\end{aligned}
$$

We can easily solve (23) using standard method of [3]. The solution of (23) is

$$
U(y, t)=y N\left(\bar{d}_{1}\right)-K N\left(\bar{d}_{2}\right) .
$$

Here

$$
\begin{aligned}
& \bar{d}_{1}=\frac{\ln \frac{y}{K}+\frac{1}{2} \sigma^{2}(t, T)}{\sigma(t, T)}, \quad \bar{d}_{2}=\bar{d}_{1}-\sigma(t, T), \\
& \sigma^{2}(t, T)=\int_{t}^{T}\left|\Sigma_{1}(u)-\Sigma_{2}(u)-\sigma_{3}\right|^{2} d u .
\end{aligned}
$$

Considering (18), then we have

$$
\sigma^{2}(t, T)=\int_{t}^{T}\left|B_{1}(u, T) \sigma_{1}-B_{2}(u, T) \sigma_{2}+\sigma_{3}\right|^{2} d u .
$$


Return to the original variables $V, p_{1}(t, T), p_{2}(t, T), F$, then we have the price of European call foreign currency option:

$$
V\left(p_{1}, p_{2}, F, t\right)=p_{2}\left(t, r_{2}, T\right) F N\left(d_{1}\right)-K p_{1}\left(t, r_{1}, T\right) N\left(d_{2}\right),
$$

where

$$
d_{1}=\frac{\ln \frac{p_{2}\left(t, r_{2}(t), T\right) \cdot F(t)}{p_{1}\left(t, r_{1}(t), T\right) \cdot K}+\frac{1}{2} \sigma^{2}(t, T)}{\sigma(t, T)}, \quad d_{2}=d_{1}-\sigma(t, T) .
$$

Note: The formula (24) coincides with the pricing formula in [6].

\section{The Invariance of the Form in Parabolic Equation}

In fact, the invariance of the form of Black-Scholes equations is based on the invariance of the form in parabolic equation under a change of variables with the linear combination of variables. Using the theorem 1 and the change of variable $x_{i}=\ln S_{i}$, we can easily get a transformation under which the form of parabolic equation is not changed and the dimension is reduced.

As shown in [3], the change of variable $x_{i}=\ln S_{i}(i=0,1, \cdots, n)$ transforms the equation (11) into a parabolic equation and we have the diagram:

$$
\begin{aligned}
& \text { (1) } \underset{x=\ln S}{\overleftrightarrow{\longrightarrow}} \frac{\partial V}{\partial t}+\frac{1}{2} \sum_{i, j=0}^{n} a_{i j} \frac{\partial^{2} V}{\partial x_{i} \partial x_{j}}+\sum_{i=0}^{n}\left(r-q_{i}-\frac{a_{i i}}{2}\right) \frac{\partial V}{\partial x_{i}}-r V=0 \\
& \text { I (2) } \\
& \text { (4) } \underset{y=\ln z}{\longleftrightarrow} \frac{\partial V}{\partial t}+\frac{1}{2} \sum_{i, j=1}^{n} \bar{a}_{i j} \frac{\partial^{2} V}{\partial y_{i} \partial y_{j}}+\sum_{i=1}^{n}\left(r-\bar{q}_{i}-\frac{\bar{a}_{i i}}{2}\right) \frac{\partial V}{\partial y_{i}}-r V=0
\end{aligned}
$$

where new change of variables $\mathbf{y}=T \mathbf{x}$ is given by

$$
\begin{aligned}
& y_{1}=\alpha_{0} x_{0}+\alpha_{1} x_{1}, \\
& y_{i}=x_{i}, \quad i=2, \cdots i=2, \cdots, n .
\end{aligned}
$$

This change of variables reserve the form of parabolic equation and reduce the number of space variables.

\section{Conclusions}

Multi-dimensional Black-Scholes equations have the form invariance under the change of variables product (2) and its space dimension is reduced under the change of variables.

In the pricing problems of financial derivatives described as a terminal value problem for multi-dimensional Black-Scholes equation, if its expiry payoff has the combination of variables such as (2), then the space dimension can be reduced.

In some pricing problems of interest rate derivative that have three or more risk resources and are not described by Black-Scholes equations (for example, [6]), the space dimension can be reduced by two or more; the main reason is that the pricing problem is described as a simillar form of multi-dimensional Black-Scholes equation when we consider the interest 
rate derivative as a risk free zero coupon bond derivative and its expiry payoff function has not only a combination of variables but also homogeneity on the new group variables.

The method of considering interest rate derivatives as no coupon bond derivatives is still effective in any interest rate models satisfied the assumptions "(i) the volatility of short rate $r$ does not depend on $r$, (ii) the price of zero coupon bond follows geometric Brown motion (see (18)), (iii) short rate $r$ is a deterministic function of the price of zero coupon bond (see (17))". For examples, Vasicek, Ho-Lee and Hull-White models satisfy all these properties. For counter examples, HJM model satisfies (i) and (ii) but does not satisfy (iii); CIR model satisfies (ii) and (iii) but does not satisfy (i), so the resulting equations (22) and (23) after change of variables have the coefficients $\Sigma_{1}, \Sigma_{2}$ that still depend on $r$ or $p_{1}$ and $p_{2}$.

If an asset $F$ that depends on the short rate satisfies the above three assumptions, then we can consider interest rate derivatives as $F$ derivatives and use Black-Scholes equations. For a counter example, if $r$ is stochastic and satisfies (i), $B$ is bank account, that is

$$
B(t)=\exp \int_{0}^{t} r(u) d u,
$$

then $B$ satisfies (ii) but it does not satisfy (iii), so we cannot consider interest rate derivatives as $B$ derivatives.

\section{References}

[1] Benninga, S., Bjrk, T. and Wiener, Z. On the Use of Numeraires in Option Pricing. The Journal of Derivatives. Winter 2002. 10(2): 1-16.

[2] Garman, M. and Kohlhagen, S. Foreign currency option values. Journal of international money and Finance. 1983. 2(3): 231-237.

[3] Jiang, L. Mathematical modeling and methods of option pricing. Beijing: Higher education press. 2003. chap. 7 (in Chinese), English translation: Jiang, Li-shang. Mathematical modeling and methods of option pricing. Singapore: World Scientific. 2005.

[4] O, H., Ro, Y. and Wan, N. The Use of Numeraires in Multi-dimensional Black-Scholes Partial Differential Equations. Working Paper. Tong-ji University, Department of Applied Mathematics. 2005. http://ssrn.com/abstract=731544. DOI: $10.2139 /$ ssrn.731544 (in Chinese), English translation: arXiv 1310.8296[q-fin-PR].

[5] Wilmott, P. Derivatives, The theory and practice of financial engineering. New York: John Wiley \& Sons, Inc.1998.

[6] Xu, G. Analysis of Pricing European Call Foreign Currency Option Under the Vasicek Interest Rate Model. Journal of Tongji University(Natural Science). 2006. 34(4): 552556 . 